Virginia Commonwealth University vCU Scholars Compass

2013

\title{
On applications and limitations of one-dimensional capillarity formulations for media with heterogeneous wettability
}

T. M. Bucher

Virginia Commonwealth University

H. Vahedi Tafreshi

Virginia Commonwealth University, htafreshi@vcu.edu

Follow this and additional works at: http://scholarscompass.vcu.edu/egmn_pubs

Part of the Mechanical Engineering Commons, and the Nuclear Engineering Commons

Bucher, T.M., and Tafreshi, H.V. On applications and limitations of one-dimensional capillarity formulations for media with heterogeneous wettability. Applied Physics Letters, 102, 241606 (2013). Copyright (C 2013 AIP Publishing LLC.

\section{Downloaded from}

http://scholarscompass.vcu.edu/egmn_pubs/14

This Article is brought to you for free and open access by the Dept. of Mechanical and Nuclear Engineering at VCU Scholars Compass. It has been accepted for inclusion in Mechanical and Nuclear Engineering Publications by an authorized administrator of VCU Scholars Compass. For more information, please contact libcompass@vcu.edu. 


\title{
On applications and limitations of one-dimensional capillarity formulations for media with heterogeneous wettability
}

\author{
T. M. Bucher and H. Vahedi Tafreshi ${ }^{\mathrm{a})}$ \\ Department of Mechanical and Nuclear Engineering, Virginia Commonwealth University, \\ Richmond, Virginia 23284-3015, USA
}

(Received 14 April 2013; accepted 31 May 2013; published online 18 June 2013)

\begin{abstract}
Force-balance-based one-dimensional algebraic formulations that are often used in characterizing the capillarity of a multi-component system (e.g., predicting capillary height rise in porous media) are discussed. It is shown that such formulations fail to provide accurate predictions when the distribution of wetting (or non-wetting) surfaces is not homogeneous. A more general mathematical formulation is suggested and used to demonstrate that for media with heterogeneous wettability, hydrophilic (or hydrophobic) surfaces clustered in groups will have less contribution to the overall capillarity of the system. (C) 2013 AIP Publishing LLC. [http://dx.doi.org/10.1063/1.4811167]
\end{abstract}

Capillarity was first explored by measuring vertical fluid rise $z$ in a capillary tube of fixed diameter $d_{\text {cap }}$, and relating it to the physical properties of the tube and fluid, eventually culminating in the Young-Laplace equation, or for height rise, Jurin's law ${ }^{1}$

$$
z=\frac{4 a^{2} \cos \theta_{c a p}}{d_{c a p}}
$$

where $\theta_{\text {cap }}$ is the liquid-solid contact angle and $a=\sqrt{\sigma / \rho g}$ is equal to capillary length scale. $\sigma, \rho$, and $g$ represent surface tension, density, and gravitational acceleration, respectively. The effect of capillarity is significant when $d_{\text {cap }}$ is less than capillary length $a{ }^{2}$ Later, a dynamic model for 1-D Poiseuille flow to predict fluid height rise in a vertical capillary tube was devised, ${ }^{3,4}$ the static (equilibrium) component of which becomes Eq. (1). This model has been adapted for modeling fluid transport in porous media as a series of aligned capillary tubes of a single equivalent diameter. ${ }^{5}$ The same basis for derivation was later revisited by Marmur, ${ }^{6}$ in which a model for predicting the radial horizontal spread of a fluid from an infinite reservoir of radius $R_{O}$ was developed, and later validated for spread in sheet papers. ${ }^{7}$ A modified form of Marmur's equation was then presented in Ref. 8 as

$$
\left(\frac{R}{R_{0}}\right)^{2}\left(\ln \frac{R}{R_{0}}-\frac{1}{2}\right)+\frac{1}{2}=\frac{\sigma d_{c a p} \cos \theta_{c a p}}{12 \mu R_{0}^{2}} t .
$$

Equation (2), or any such equation derived based on a capillary-tube representation of a multi-component fibrous medium (medium having more than one fiber diameter and/ or wall-contact angle), requires single numeric values for the diameter and contact angle of the capillary tube, $d_{c a p}$ and $\theta_{\text {cap }}$. In this work, we discuss the applicability of the 1-D force-balance approach for obtaining $d_{\text {cap }}$ and $\theta_{\text {cap }}$ for multicomponent fibrous media using fiber bundles (media

\footnotetext{
a) Author to whom correspondence should be addressed. Electronic mail: htafreshi@vcu.edu. Tel.: 804-828-9936. Fax: 804-827-7030. URL: http:// www.people.vcu.edu/ htafreshi. Present address: Virginia Commonwealth University, Richmond, Virginia, USA.
}

comprised of highly aligned fibers), as a special-case representation of a concept that can generally be applied to all systems in which capillary pressure is a driving mechanism, such as biological systems and capillary pumps. ${ }^{9-11}$

Let us consider a square array of four fibers as a unit cell in a bundle of vertically aligned fibers, as shown from overhead in Figure 1. Each fiber may be one of two materials, each of which with its own contact angle $\theta$ and fiber diameter $d$. The meniscus height $z$ in such a medium can be described as

$$
z=f\left(\alpha, d_{1}, d_{2}, \ldots, \theta_{1}, \theta_{2}, \ldots, n_{1}, n_{2}, \ldots\right),
$$

where $\alpha$ is equal to solid volume fraction (SVF) of the medium, and $d_{i}, \theta_{i}$, and $n_{i}$ are, respectively, diameter, liquid-solid contact angle, and number fraction (between 0 and 1) for the $i$ th fiber type. We can derive an expression for meniscus height rise based on the balance of forces across the meniscus as shown in Figure 1 , with $F_{g}$ and $F_{\sigma}$ being gravity and capillary forces, respectively

$$
A \rho g z_{\min }=C \sigma \cos \theta
$$

where $A$ and $C$ are total available fluid cross-sectional area and liquid-air-fiber interface perimeter, and $z_{\text {min }}$ refers to the height of the bottom of the meniscus. Expanded in terms of Eq. (3) for a unit cell with two different fiber types, Eq. (4) becomes

$$
z_{\min }=\frac{4 \sigma\left(n_{1} d_{1} \cos \theta_{1}+n_{2} d_{2} \cos \theta_{2}\right)}{\rho g(1 / \alpha-1)\left(n_{1} d_{1}^{2}+n_{2} d_{2}^{2}\right)} .
$$

It is worth mentioning that this equation simplifies to that of Princen ${ }^{12}$ in the special case of one single fiber size and contact angle. From Eqs. (1) and (5), one obtains

$$
\frac{\cos \theta_{\text {cap }}}{d_{\text {cap }}}=\frac{\left(n_{1} d_{1} \cos \theta_{1}+n_{2} d_{2} \cos \theta_{2}\right)}{(1 / \alpha-1)\left(n_{1} d_{1}^{2}+n_{2} d_{2}^{2}\right)} .
$$

Examining Eq. (6) reveals that this equation is in fact comprised of two more familiar equations, a weighted averaging of cosines of the two contact angles for the cosine of 


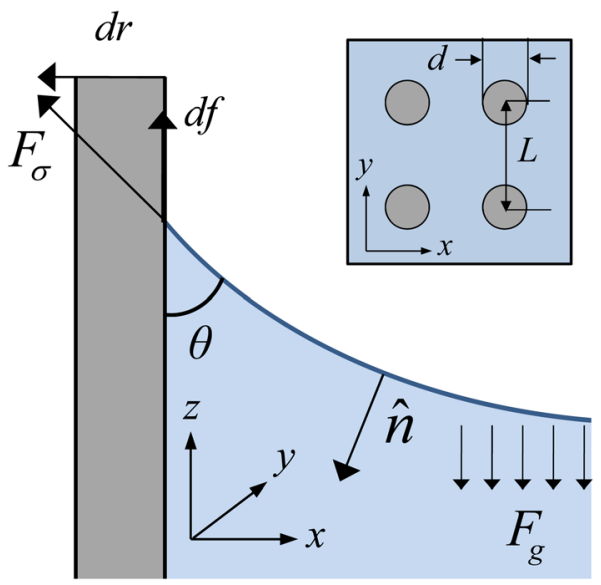

FIG. 1. Free-body diagram of the balance of forces along a fiber in a medium (overhead view of unit cell shown in upper right).

equivalent contact angle $\theta_{\text {cap }}$, and classical hydraulic diameter for equivalent capillary diameter $d_{\text {cap }}$, i.e.,

$$
\begin{gathered}
\cos \theta_{\text {cap }}=\frac{n_{1} d_{1}}{n_{1} d_{1}+n_{2} d_{2}} \cos \theta_{1}+\frac{n_{2} d_{2}}{n_{1} d_{1}+n_{2} d_{2}} \cos \theta_{2}, \\
d_{\text {cap }}=4 \frac{A}{C}=\left(\frac{1}{\alpha}-1\right) \frac{n_{1} d_{1}^{2}+n_{2} d_{2}^{2}}{n_{1} d_{1}+n_{2} d_{2}} .
\end{gathered}
$$

One therefore can use Eqs. (7) and (8) together with Eq. (1) to predict a fluid's height rise, or Eq. (2) (among others) to predict a fluid's radial horizontal spread in multi-component media. To better examine this, we consider a more accurate 2-D force-balance approach that can produce the local height of the air-water interface at every $(x, y)$ point in the media. ${ }^{13-15}$ The approach begins with the Young-Laplace equation for an arbitrary geometry subject to capillary forces

$$
F(x, y)=a^{2}(\vec{\nabla} \cdot \hat{n}),
$$

where $F(x, y)$ is the local height of the meniscus, and $\hat{n}$ is its unit normal vector. Defining a function $G(x, y, z)=$ $F(x, y)-z$ for which $G=0$ along the meniscus, we obtain the unit normal vector as $\hat{n}=\nabla G /|\nabla G|$, and Eq. (9) becomes

$$
\begin{aligned}
F\left(F_{x}^{2}+F_{y}^{2}+1\right)^{3 / 2}= & a^{2}\left(\left(1+F_{y}^{2}\right) F_{x x}+\left(1+F_{x}^{2}\right) F_{y y}\right. \\
& \left.-2 F_{x} F_{y} F_{x y}\right)
\end{aligned}
$$

where the subscripts of $x$ and $y$ represent respective partial derivates of $F(x, y)$. This equation is solved using a finite element method (the FLEXPDE software from PDESolutions, Inc.) with a fiber boundary condition of $|\nabla F|_{f i b}=\cot \theta$, and periodic boundary conditions on the lateral sides of the domain. A minimum inter-fiber distance of $d_{f} / 3$ was enforced in our random structures to avoid additional capillary effects that occur in corners with small angles. ${ }^{2,16}$

Figure 2 shows an example of local height values obtained from solving Eq. (10), in which a medium with $\alpha=$ 0.15 and $d_{f}=10 \mu \mathrm{m}$ is considered. A bimodal contact-angle distribution with half the fibers having $\theta_{1}=45^{\circ}$, and the other half $\theta_{2}=85^{\circ}$ is considered. The $z$-axis in the contour

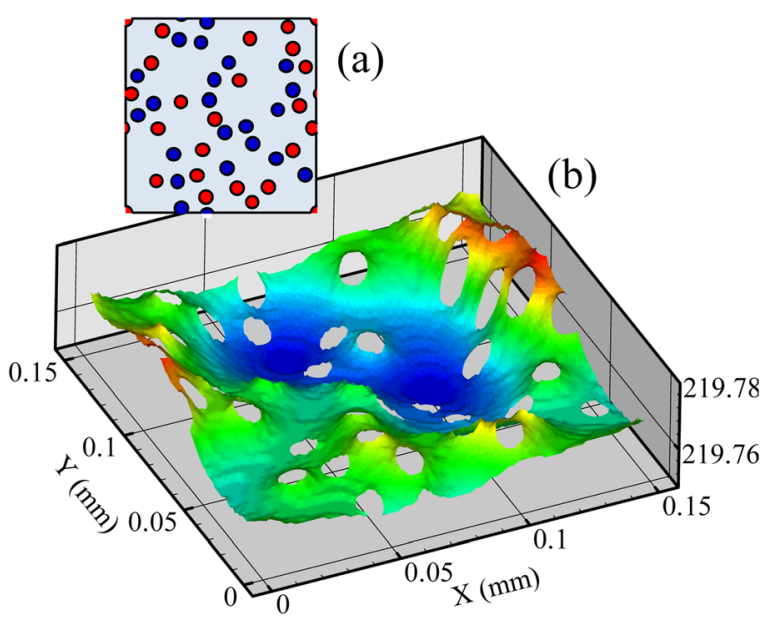

FIG. 2. (a) Sample computational domain with random fiber spacing. $\mathrm{SVF}=0.15, d=10 \mu \mathrm{m}, \theta_{1}=45^{\circ}$, and $\theta_{2}=85^{\circ}$ (evenly distributed); (b) contour plot of local meniscus height $(\mathrm{mm})$. Equation (4) predicts height rise of $208.5 \mathrm{~mm}$ for this medium, about $5 \%$ difference from Eq. (10).

plot shows local height in $\mathrm{mm}$. We have compared predictions of an ensemble of 1-D and 2-D force-balance calculations for media with SVFs from 5-15\%, fiber diameters from $5-25 \mu \mathrm{m}$, and contact angles from $25^{\circ}-85^{\circ}$, allowing both for unimodal and bimodal fiber size and component distributions. We observed good agreement (within 10\% margin of error) between the minimum meniscus height of Eq. (5) and the average meniscus height of Eq. (10) for all microstructure-property combinations considered. We performed our calculations not only on unit cells (Figure 1), but also on larger domains in which a greater number of fibers are distributed randomly (Figure 2(a)).

In Figure 3, we illustrate the overall effect of bimodality on capillarity, plotting height rise as a function of number fraction of coarse fibers $n_{c}$. For all structures, we considered an SVF 0.1 and a fine fiber diameter of $10 \mu \mathrm{m}$ (the parameter $R_{c f}$ refers to the size ratio between coarse and fine fibers). It can be seen that capillarity reduces sharply with the introduction of larger fibers, and more so the larger the coarse fibers. This is because with the available solid volume being consolidated into fewer larger fibers, the capillary force is left with less solid surface area over which to act. This is also the

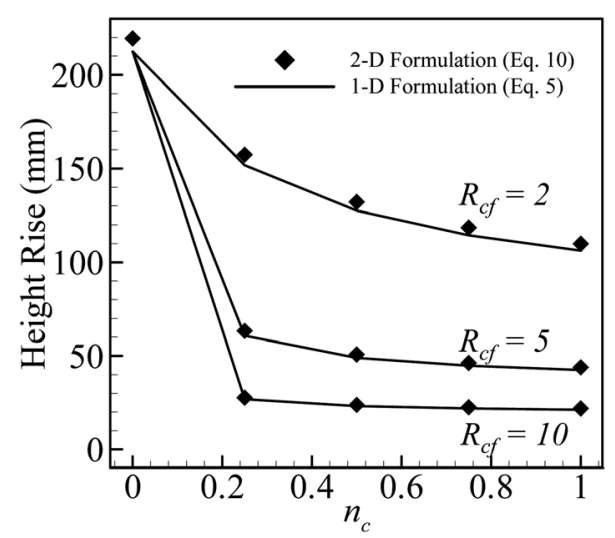

FIG. 3. Capillary height rise as a function of coarse fiber fraction $n_{c}$. Solutions for both 1-D minimum height (Eq. (5)) and average 2-D height $\bar{F}$ (Eq. (10)) are shown. For all structures, SVF $=0.1, d_{f}=10 \mu \mathrm{m}$, and $\theta=50^{\circ}$. $R_{c f}$ is the size ratio between coarse and fine fibers. 
case for structures containing only one larger fiber size (Figure 3 when $n_{c}=1$ ).

Until this point, we have only explored structures in which parameters are distributed either orderly (unit cells) or in which fibers of given properties are distributed randomly, though homogeneously. In reality, it is conceivable to have conditions otherwise. Consider a square array of equal-sized fibers in which two contact angles are distributed in equal proportions (or as close as achievable). Different fibers are each hydrophilic and hydrophobic by the same degree. Now consider two cases in which the hydrophobic fibers are either distributed uniformly (Figure 4(a)), or clustered together in the middle (Figure 4(b)). For Figures 4(a) and 4(b), we solved Eq. (10) on two square arrays of fibers. For both figures, SVF is 0.1 , and fiber diameter is $10 \mu \mathrm{m}$. For Figure $4(\mathrm{a})$, the structure is a $6 \times 6$ array of fibers with properties alternating from fiber to fiber, $\theta_{1}=60^{\circ}$ and $\theta_{2}=120^{\circ}$. In this case, the two effects cancel, and the overall height rise is zero according to Eq. (5). Figure 4(b) is a $7 \times 7$ array of fibers. In this case, twenty-four fibers of $\theta_{1}=60^{\circ}$ line the boundary, and twenty-five others of $\theta_{2}=120^{\circ}$ are clustered inside. As can be seen, it is clear that the hydrophilic fibers dominate overall behavior, with a height rise on the order of $6.5 \mathrm{~mm}$.

This effect can be explained considering that the computational domain shown in Figure 4(b) represents a large medium in which isolated hydrophobic fibers are placed in a matrix of hydrophilic fibers with the same number density.
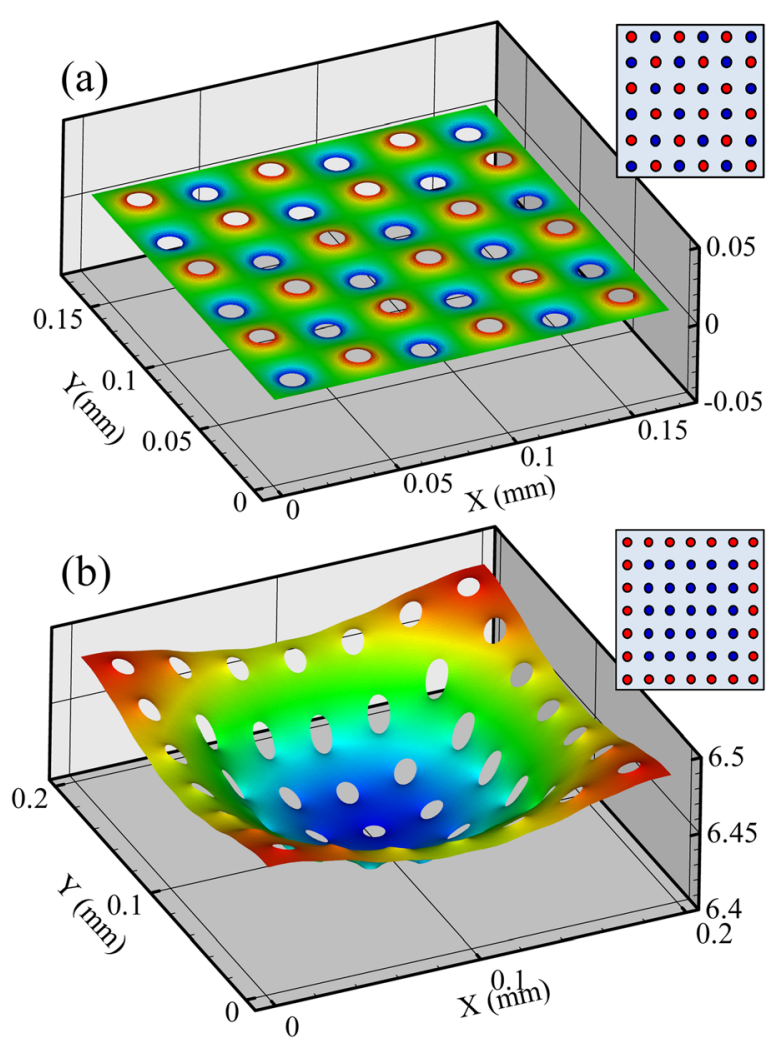

FIG. 4. Contour plot of local height $F$ for a square array of fibers in which: (a) contact angles $\theta_{1}=60^{\circ}$ and $\theta_{2}=120^{\circ}$ are uniformly distributed, and (b) fibers with $\theta_{1}=60^{\circ}$ line the boundaries of the structure, and those with $\theta_{2}=120^{\circ}$ are clustered in the middle. Conceptual layouts to the right of respective plots are colored with red and blue fibers for $\theta_{1}=60^{\circ}$ and $\theta_{2}=120^{\circ}$, respectively.
The results shown in Figure 4(b) indicate that the fibers in the matrix produce a collective effect to determine the wettability of the medium, even though their number fraction in the media is the same as those clustered together. We also examined the case opposite to Figure 4(b), switching the contact angles of the constituent fibers, and noticed an equally strong overall hydrophobic effect (not shown for brevity). For each case shown in Figures 4(a) and 4(b), Eq. (5)—or more generally, Eq. (4)—incorrectly predicts a zero height rise, as equal proportions of hydrophilic and hydrophobic surface area cancel each other out.

Another example showing the 1-D force-balance equations failing to predict the wettability of a system is the family of grooved multi-component fibers (i.e., fibers made up of more than one polymer) sometimes used for efficient fluid transport in absorbent media. ${ }^{17}$ Here, we considered tipped four-lobed rosettes, where the tip polymer is different from that of the core. In this case (Figure 5(a)), the unit cell consists of a single fiber comprised of two orthogonal ellipses, the major and minor diameters of which are arbitrarily chosen to be 15 and $3 \mu \mathrm{m}$. Both the tips and core have equal surface areas. The tips of the fiber have one contact angle $\left(\theta_{1}=50^{\circ}\right)$, while the core portion has an equal opposite contact angle with respect to the neutral wetting condition $\left(\theta_{2}=130^{\circ}\right)$. As can be seen, there is clearly a dominant hydrophilic effect present in the structure. This occurs for the same reason that the bordering (matrix) fibers in Figure 4(b) dominate the overall performance. That is, the tips of the fiber are able to interact with the tips of neighboring fibers, allowing their collective effect to form a hydrophilic network throughout the medium. The core portion is isolated not only from other cores but even a single quadrant on a fiber is isolated from other quadrants of the same fiber. Again, the opposite contact-angle configuration produces the opposite (hydrophobic) effect (not shown for brevity). As mentioned earlier, Eq. (4) fails to capture this effect, and incorrectly predicts a zero height rise, as equal proportions of hydrophilic and hydrophobic surface area cancel mathematically. Thus, in situations in which capillary pressure

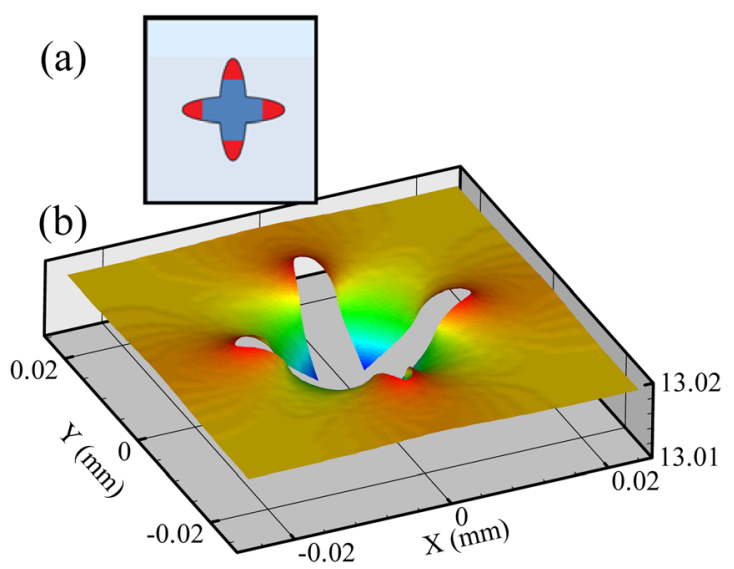

FIG. 5. (a) Unit cell containing one four-lobed rosette-shaped fiber. Domain size corresponds to an SVF of 0.1. Fiber is constructed using two orthogonal ellipses with major and minor diameters of $15 \mu \mathrm{m}$ and $3 \mu \mathrm{m}$. Blue and red regions represent fiber portions with opposing contact angles. (b) 3-D contour plot of capillary height rise in the structure. The outer portion of the fiber (tips) possesses a $\theta$ of $50^{\circ}$, while the inner portion (core) possesses a $\theta$ of $130^{\circ}$. 


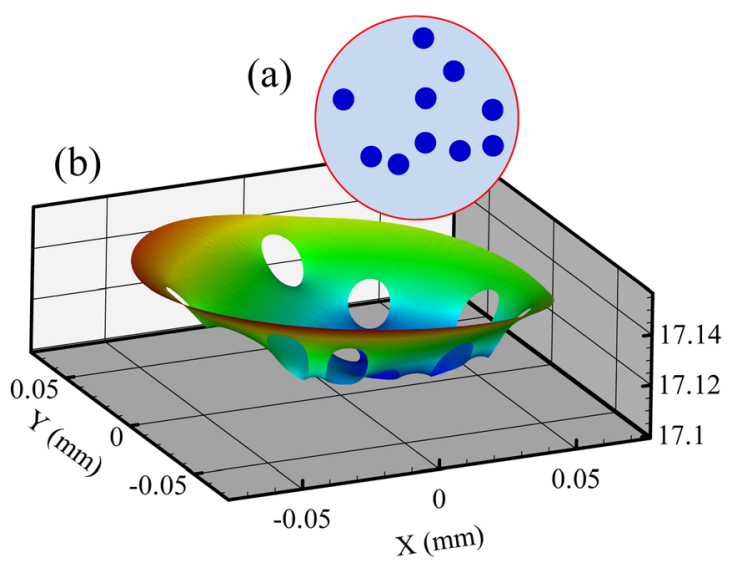

FIG. 6. (a) Vertical capillary domain (red outline) with ten aligned fibers running through its interior (dark blue); (b) contour plot of local height $F$. $d_{\text {cap }}=140 \mu \mathrm{m}, \theta_{\text {cap }}=60^{\circ}, d_{f}=14 \mu \mathrm{m}$, and $\theta_{f}=120^{\circ}$.

must be determined using a 2-D approach (Figures 4(b) and 5(b), for instance), Eqs. (7) and (8) can no longer be used to determine the appropriate $\theta_{\text {cap }}$ and $d_{c a p}$ as described earlier.

Recall that Eq. (2) provides a means of extending the Washburn approach to capillarity to the case of radial spread in which case the method presented in this work can be applied to predict the rate of fluid absorption in multicomponent media using Eqs. (7) and (8). However, in media with heterogeneously distributed wettability, an alternative approach is needed. For instance, one can use an equation given in Ref. 8 such as

$$
\left(\frac{R}{R_{0}}\right)^{2}\left(\ln \frac{R}{R_{0}}-\frac{1}{2}\right)+\frac{1}{2}=\frac{2 k p_{c}}{(1-\alpha) \mu R_{0}^{2}} t
$$

where $p_{c}=\rho g \bar{F}$ is the capillary pressure, with $\bar{F}$ representing the average height rise to be obtained from solving Eq. (10), and $k$ is the permeability constant of the medium. There are numerous expressions reported for predicting the permeability of fibrous structures made of circular fibers (e.g., Refs. 18-20). However, for non-circular fibers, a numerical solution of Stokes' equation $\partial p / \partial z=\mu \nabla^{2} u$ similar to Ref. 21 may be necessary. The pressure drop obtained from such a calculation can then be used in Darcy's law $\bar{u}=(-k A / \mu)(\partial p / \partial z)$ to obtain the permeability constant $k(u$ and $\bar{u}$ are local and area-averaged velocities, respectively).

Besides porous media applications, breakdown of the 1-D capillarity formulation is equally important in applications involving fluid transport in capillary tubes with heterogeneous internal structures for micro-fluidics, drug delivery, or biological applications among others. ${ }^{22-26}$ Consider a capillary tube made of one material filled with fibers of made of another (Figure 6(a)). ${ }^{27-29}$ The interplay between the tube and the fibers can be used to engineer a desired capillary effect using available materials for such applications. Figure 6(b) illustrates the solution of Eq. (10) for a capillary tube in $\mathrm{mm}$ containing ten fibers, in which the surface area of the tube equals that of the fibers. As can be seen, the tube wall still dominates the overall behavior (where 1-D equations would predict a zero height rise). It is worth mentioning that varying the tube size or the number of internal fibers, while keeping the tube and fiber surface areas equal, indicates that addition of more fibers further reduces the overall height rise, as the wettability tends to become more homogenous with increasing the number of fibers.

In summary, the 1-D capillarity formulations can be applied to multi-component systems (Eqs. (1), (2), (7), and (8)) to predict the rate of fluid transport as long as the wettability is distributed homogenously. However, when the distribution of surfaces with different wettability is not homogenous, a more rigorous mathematical approach should be taken that requires the solution of Eq. (10). It was shown in particular that when the fibers of a given type are clustered together, their influence on the overall behavior is weakened.

The authors would like to acknowledge the financial support for this work from National Science Foundation CMMI program Grant No. 1029924.

${ }^{1}$ J. Jurin, Philos. Trans. R. Soc. London 30, 739-747 (1919).

${ }^{2}$ A. Ponomarenko, D. Quére, and C. Clanet, J. Fluid Mech. 666, 146-154 (2011).

${ }^{3}$ R. Lucas, Kolloid-Z. 23, 15 (1920).

${ }^{4}$ E. Washburn, Phys. Rev. 17, 273 (1921).

${ }^{5}$ F. A. L. Dullien, Porous Media Fluid Transport and Pore Structure, 2nd ed. (Academic Press, San Diego, 1992).

${ }^{6}$ A. Marmur, J. Colloid Interface Sci. 124, 301-308 (1988).

${ }^{7}$ Danino and Marmur, J. Colloid Interface Sci. 166, 245-250 (1994).

${ }^{8}$ Hyvaluoma, P. Raiskinmaki, A. Jasberg, A. Kaponen, M. Kataja, and J. Timonen, Phys. Rev. E 73, 036705 (2006).

${ }^{9}$ M. Zimmerman, H. Schmid, P. Hunziker, and E. Delamarche, Lab Chip 7, 119-125 (2007).

${ }^{10}$ B. A. Malouin, Jr., M. J. Vogel, J. D. Olles, L. Cheng, and A. H. Hirsa, Lab Chip 11, 393-397 (2011).

${ }^{11}$ P. Koumoutsakos, I. Pivkin, and F. Milde, Annu. Rev. Fluid Mech. 45, 325-355 (2013).

${ }^{12}$ H. M. Princen, J. Colloid Interface Sci. 30, 359 (1969).

${ }^{13}$ B. Emami, H. V. Tafreshi, M. Gad-el-Hak, and G. C. Tepper, Appl. Phys. Lett. 98, 203106 (2011).

${ }^{14}$ B. Emami, H. V. Tafreshi, M. Gad-el-Hak, and G. C. Tepper, Appl. Phys. Lett. 100, 013104 (2012).

${ }^{15}$ B. Emami, H. V. Tafreshi, M. Gad-el-Hak, and G. C. Tepper, J. Appl. Phys. 111, 064325 (2012).

${ }^{16}$ Weislogel, J. Fluid Mech. 709, 622-647 (2012).

${ }^{17}$ I. M. Hutten, Handbook of Nonwoven Filter Media (Elsevier Ltd., 2007).

${ }^{18}$ S. A. Hosseini and H. V. Tafreshi, Chem. Eng. Sci. 65, 2249-2254 (2010).

${ }^{19}$ M. A. Tahir and H. V. Tafreshi, Phys. Fluids 21, 083604 (2009).

${ }^{20}$ A. Tamayol and M. Bahrami, ASME Trans. J. Fluid Eng 132, 114502 (2010).

${ }^{21}$ S. A. Hosseini and H. V. Tafreshi, Powder Technol. 212, 425-431 (2011).

${ }^{22}$ K. Madhaiyan, R. Sridhar, S. Sundarrajan, J. R. Venugopal, and S. Ramakrishna, Int. J. Pharm. 444, 70-76 (2013).

${ }^{23}$ D. Halpern, H. Fujioka, S. Takayama, and J. B. Grotberg, Respir. Physiol. Neurbiol. 163, 222-231 (2008).

${ }^{24}$ H. Yoshida, M. Matsusaki, and M. Akashi, Adv. Funct. Mater. 23, 1736-1742 (2013).

${ }^{25}$ J. D. Olles, M. J. Vogel, B. A. Malouin, Jr., and A. H. Hirsa, Opt. Express 19, 19399 (2011).

${ }^{26}$ C. Popa, Y. Okayasu, K. Katsumata, T. Isobe, N. Matsushita, A. Nakajima, T. Kurata, and K. Okada, J. Mater. Sci. 48, 941-947 (2013).

${ }^{27}$ Y. Hu, C. Song, and G. Li, J. Chromatogr. A 1263, 21-27 (2012).

${ }^{28}$ Y. Saito, M. Imaizumi, T. Takeichi, and K. Jinno, Anal. Bioanal. Chem. 372, 164-168 (2002).

${ }^{29}$ Y. Saito, Y. Nakao, M. Imaizumi, T. Takeichi, Y. Kiso, and K. Jinno, Fresenius' J. Anal. Chem. 368, 641-643 (2000). 